\title{
Albrecht von Hallers Korrespondenz und ihre Erschließung
}

Von Urs Boschung

ZuSAMMENFASSUNG

Albrecht von Hallers Briefsammlung spiegelt für die Zeit von 1728 bis 1777 das wissenschaftliche und gelehrte Leben Europas wider. 12260 Briefe von 1114 Korrespondenten bewahrt die Burgerbibliothek Bern, nach den Angaben des Verzeichnisses von Thormann von 1933/35. Zur Klärung der Ausgangslage eines größeren Projekts zur wissenschaftlichen und archivalischen Erschließung des Haller-Nachlasses wird das Verzeichnis von Thormann kurz analysiert und eine Liste der bisher publizierten Haller-Briefwechsel zusammengestellt.

\section{Einleitung}

Die Bedeutung Albrecht von Hallers für Medizin und Biologie, Literatur und Philosophie, Politik und Gesellschaft des 18. Jahrhunderts ist unbestritten. Historiker aller Richtungen haben sich mit ihm immer wieder auseinandergesetzt, und dies wird auch in Zukunft so bleiben. Die Grundlage dafür sind die gedruckten Werke, und noch wichtiger, die Manuskripte und Briefe, schließlich die Arbeiten der historischen Vorgänger.

Seit Ludwig Hirzel 1882 seine immer noch unentbehrliche Biographie Hallers ${ }^{1}$ vorgelegt hat, ist zwar vieles erarbeitet und erschlossen worden, namentlich durch K.S. Guthke auf germanistischem, durch E. Hintzsche (1900-1975) auf medizinhistorischem Gebiet. Die Spezialisierung der Wissenschaft hat jedoch auch dazu geführt, daß Haller nur selten in seiner Universalität betrachtet wird. Ein interdisziplinärer Ansatz wird zudem dadurch erschwert, daß die Primärquellen der Haller-Forschung sehr umfangreich und nach wie vor nur partiell erschlossen und kritisch aufgearbeitet sind und daß die Sekundärliteratur kaum noch überblickbar ist.

Was in erster Linie not tut, ist eine wissenschaftlich-kritische Inventarisierung der Quellen ${ }^{2}$ sowie eine neue Bibliographie Hallers und der Sekun- 
därliteratur. Parallel zu dieser Bestandesaufnahme sollte eine chronikalische Darstellung seines Lebens und Werks erarbeitet werden.

Nachfolgend werden einige Notizen und Überlegungen über den Stand der Erschließung von Hallers Korrespondenz vorgelegt und am Schluß ein Projekt skizziert, dessen Realisierung in Bern seit einiger Zeit vorbereitet wird.

\section{Hallers Briefsammlung}

Als nach Albrecht von Hallers Tod sein Besitz an Büchern und Handschriften an Joseph II. verkauft wurde und nach Oberitalien gelangte, war ein wertvoller Teil von dieser Dislokation ausgenommen, nämlich die Sammlung der an Haller gerichteten Briefe. Sie gehören heute mit den 1929 aus Italien zurückgekehrten Manuskripten zum wichtigsten Bestand des Haller-Nachlasses der Berner Burgerbibliothek ${ }^{3}$.

Hallers Sohn Gottlieb Emanuel (1735-1786) verfaßte zur Briefsammlung, die 1792 mit seinem Nachlaß in die Stadtbibliothek Bern gelangte, ein handschriftliches Register ${ }^{4}$, das heute von besonderem Wert ist, da es mit der Liste von 1209 Korrespondenten und 13202 Briefen offenbar den ursprünglichen Bestand wiedergibt.

Als Dr. Franz Thormann 1935 ein neues Verzeichnis der Korrespondenten herausgab, stellte er im Vergleich mit dem alten Inventar fest, daß viele gerade der wichtigsten Schriftstücke verschwunden waren ${ }^{5}$. Bekannt war, daß von Hallers Sohn Rudolf Emanuel (1747-1833) mehreres allzu Intime entfernt, anderes von Autographenjägern entwendet worden war.

Seither hat die Burgerbibliothek Bern mit großer Aufmerksamkeit im Handel auftauchende Halleriana, besonders auch Briefe von Haller, gesammelt und so einiges zurückerwerben können, was früher schon zum eigenen Bestand gehörte; manches konnte auch in auswärtigen Sammlungen nachgewiesen und wenigstens in Kopie beschafft werden ${ }^{6}$.

\subsection{Aufbau der Sammlung}

Haller hat die von ihm aufgehobenen Briefe schon zu Lebzeiten einbinden lassen, und zwar in drei, zeitlich zum Teil parallel laufenden Serien ${ }^{7}$. Durch die starke Benützung haben seither zahlreiche Briefbogen, besonders die großformatigen, zusammengefalteten, Schaden genommen. So wurde unter 
der Leitung von Herrn Dr. Hans Haeberli in den letzten Jahren der gesamte Bestand vom Atelier der Stadt- und Universitätsbibliothek Bern (Hr. Erwin Oberholzer) restauriert, was eine Auflösung der Briefbände bedingte. Damit war der Weg frei zur Neuordnung nach Korrespondenten, wie sie bis im Herbst 1989 durch Herrn Dr. phil. Emil Erne vollzogen wurde. So ist die Benützung, die erfahrungsgemäß meist nach Korrespondenten erfolgt, wesentlich leichter geworden; die alte chronologische Ordnung ist nach den Inventaren der einzelnen Bände immer noch feststellbar; auch ist auf jedem einzelnen Brief angegeben, in welchem Band er sich früher befand.

\subsubsection{Briefe an Haller}

Für die Briefe an Haller nennt Thormann in seinem Verzeichnis 1114 Korrespondenten mit insgesamt 12260 Briefen. Im Durchschnitt ergäben sich also 11 Briefe auf jeden Korrespondenten; nur 8 Briefschreiber ensprechen jedoch genau diesem Mittel. Tatsächlich liegt von 506 Korrespondenten $(45,5 \%)$ lediglich ein einziger Brief vor; dagegen steuern 66 Korrespondenten (5,9\%) $68 \%$ aller Briefe bei (siehe Liste im Anhang).

Tabelle 1

Briefe an Haller, Verteilung nach Korrespondenzgröße

(Anzahl Briefe je Korrespondent) (nach Thormann)

\begin{tabular}{lcccc}
\hline Briefe an Haller & \multicolumn{2}{c}{ Korrespondenten } & \multicolumn{2}{c}{ Briefe insgesamt } \\
Anzahl/Korrespondent & Anzahl & Prozent & Anzahl & Prozent \\
30 und mehr & 66 & 5,9 & 8361 & 68,1 \\
$29-20$ & 24 & 2,1 & 573 & 4,6 \\
$19-10$ & 82 & 7,3 & 1144 & 9,3 \\
$9-5$ & 133 & 11,9 & 870 & 7,0 \\
$4-3$ & 149 & 13,4 & 498 & 4,0 \\
2 & 154 & 13,8 & 308 & 2,5 \\
1 & 506 & 45,5 & 506 & 4,1 \\
Total & 1114 & $100 \%$ & 12260 & $100 \%$ \\
\hline
\end{tabular}

Obschon Thormanns Verzeichnis durchaus nicht mehr in allen Einzelheiten dem heutigen Stand der Sammlung (und der Kenntnisse) entspricht, lassen sich auch noch einige weitere allgemeine Feststellungen anfügen. 
Über die Herkunft der Briefe sagt H. Blösch im Verzeichnis von 1933/35 summarisch: «Die 1114 Korrespondenten Hallers umfassen einen großen Teil der Gelehrtenrepublik im 18. Jahrhundert von Rußland bis England, von Italien bis Schweden. ${ }^{8}$

Offensichtlich meint die Ortsangabe von Thormann nicht in erster Linie die Herkunft der einzelnen Briefe, sondern die hauptsächliche Tätigkeitsstätte des Korrespondenten, die natürlich oftmals mit dem Abgangsort übereinstimmt. Unter dieser Voraussetzung lassen sich die Korrespondenten wie folgt geographisch zuordnen:

Tabelle 2

Geographische Zuordnung von Hallers Korrespondenten (nach Thormann, z. T. modifiziert)

Land, geographischer Raum

«Deutschland»

\begin{tabular}{lc}
\multicolumn{3}{l}{ Korrespondenten } \\
Anzahl & Prozent \\
375 & 33 \\
301 & 27 \\
99 & 9 \\
82 & 7 \\
36 & 3 \\
36 & 3 \\
25 & 2 \\
21 & 2 \\
17 & 2
\end{tabular}

Schweiz

Frankreich

Italien

Niederlande

Großbritannien

Österreich-Ungarn

Rußland

Schweden

Weiter sind vertreten:

Dänemark mit 14 Korrespondenten, Polen mit 6, Spanien 4, Irland 2, Belgien 1; ohne Ortsangabe bleiben 94, d.h. $8 \%$ der Korrespondenten.

Noch schwieriger ist die Zuordnung zu einzelnen Fachdisziplinen und Berufsgruppen. Auch sagt die Berufsbezeichnung nichts aus über den Inhalt der Korrespondenz, da Haller ohne weiteres mit einem Geistlichen oder einem Offizier über Botanik, mit einem Arzt über Literatur korrespondiert.

Von Thormann ausgehend (und ihn in Einzelheiten auch ergänzend oder verändernd), lassen sich folgende Angaben machen: 
Tabelle 3

Fachliche Zuordnung der Haller-Korrespondenten

(nach Thormann, modifiziert)

\begin{tabular}{lcc}
\hline Fachliche Bezeichnung & \multicolumn{2}{c}{ Korrespondenten } \\
& Anzahl & Prozent \\
med. & 419 & 37,6 \\
- med., Arzt & 272 & 24,4 \\
- med., Prof. & 91 & 8,1 \\
anat. & 26 & 2,3 \\
physiol. & 4 & 0,3 \\
nat. & 84 & 7,5 \\
bot. & 45 & 4,0 \\
phys. & 9 & 0,8 \\
iur. & 16 & 1,4 \\
theol. & 65 & 5,8 \\
phil. & 19 & 1,7 \\
hist. & 6 & 0,5 \\
Prof. (insgesamt) & 187 & 16,8 \\
Im Staatsdienst & 44 & 3,9 \\
Adel & 23 & 2,0 \\
Militär & 16 & 1,4 \\
Verleger, Buchhändler & 16 & 1,4 \\
Illustratoren, Maler & 7 & 0,6 \\
Frauen & 39 & 3,5 \\
ohne Angabe & & 28,3 \\
\hline
\end{tabular}

Bemerkung: Bei einzelnen Personen können mehrere Angaben vorkommen.

Demnach setzt sich der Kreis von Hallers Korrespondenten zu mehr als einem Drittel aus Medizinern zusammen; die zweitgrößte Gruppe bilden die «Naturforscher», die drittgrößte die Theologen. 26\% der Briefverfasser können nach Thormann nicht eingeordnet werden. 
Weitergehende Analysen von Hallers Korrespondentennetz müssen u. a. von der Detailerschließung der Briefsammlung, von der Sichtung der Themenkreise des Inhalts und von biographischen Untersuchungen ausgehen.

\subsubsection{Briefe von Haller}

Die Gegenbriefe Hallers zur Berner Sammlung befinden sich naturgemäß zum kleinsten Teil in Bern; sie ausfindig zu machen war und ist für alle Editoren eine besonders harte Knacknuß.

Briefe von Haller besitzen in größerer Zahl

- die Zentralbibliothek Zürich (Haller an Johannes Gessner und an J. J. Bodmer);

- die Bibliothèque publique et universitaire in Genf (Haller an Charles Bonnet und an Horace-Bénédict de Saussure);

- die Universitätsbibliothek Erlangen (Haller an J. A. Beurer, G. N. Stock, C. J. Trew) ${ }^{9}$.

Die Burgerbibliothek Bern besitzt (nach Thormann)

- 447 Briefe Hallers an S. A. A. D. Tissot (Mss. hist. helv. XVIII 69 und 70);

- 155 Briefe Hallers an seinen Sohn Gottlieb Emanuel;

- 82 Briefe an 32 weitere Korrespondenten, 13 an unbekannt.

- Zudem sind heute 724 Briefe Hallers, deren Originale sich in 61 verschiedenen auswärtigen Archiven und Bibliotheken befinden, in Form von Kopien oder Mikrofilmen benützbar.

\subsection{Publikation von Einzelbriefen und Korrespondenzen}

Die wissenschaftshistorische Bedeutung seiner Korrespondenz war schon Haller bewußt. Nach dem Vorbild anderer Briefsammlungen des 16. und 17. Jahrhunderts, namentlich der Edition von Thomas Bartholin (1618-1680 $)^{10}$ veröffentlichte er in den Jahren 1773 bis 1775 in sechs Bänden 1004 lateinische Briefe seiner Freunde aus den Jahren 1727 bis 1774 ${ }^{11}$.

In der Auswahl, die am Anfang recht großzügig, im Lauf der Jahre aber immer strenger wurde, ließ sich Haller nach den Angaben im Vorwort vom «öffentlichen Nutzen» leiten. Vieles wurde weggelassen, Eigenlob sollte möglichst unterbleiben. Die Texte bat er, aus der Zeit heraus zu beurteilen. Ein besonderer Hinweis gilt der Kontroverse mit Linné, die abgedruckten 
Briefe sollen Hallers anfänglich gutes Verhältnis zu ihm belegen. Eigene Briefe widerzugeben sei ihm nicht möglich gewesen, da er weder Entwürfe noch die abgesandten Briefe vor sich habe. - Aus drucktechnischen Gründen beginnt in Band 4 eine neue Numerierung. Die Einleitung weist auf die Rückkehr nach Bern und die Jahre in Roche hin, ferner auf die wichtigste wissenschaftliche Thematik, die Irritabilitätslehre, auch auf einige Auseinandersetzungen, u. a. mit Albinus hin. In Band 6, gewissermaßen am Ende des Unternehmens, erwägt Haller nochmals Pro und Contra einer derartigen Edition. Offenbar war das Echo geteilt. Ein Trost bleibt: «Der Nützlichkeit gibt es viele Stufen; einiges schien mir von Nutzen zu sein, was anderen nicht einleuchtete.» Fehlgriffe mögen verziehen werden angesichts des unschuldigen Wunsches, öffentlichen Nutzen zu stiften. Ein Bibliographie von Hallers Schriften und ein umfangreiches Sachregister aus der Feder von P. R. Vicat (1742-1783) schließt das Werk ab.

Vom ursprünglichen Plan, nach den lateinischen auch die deutschen, englischen und italienischen Schreiben folgen zu lassen, blieb Haller nur noch Zeit, einen schmalen Band mit 100 Briefen in deutscher Sprache herauszugeben ${ }^{12}$. «Manche nützliche(n) Wahrnehmungen» sollten sie bringen, «einzelne Zergliederungen, chirurgische Operationen, Nachrichten von merkwürdigen Krankheiten, auch wol zuweilen chymische zur selbigen Zeit wenig bekannte Handgriffe und so genannte Processe.» Literarisches steht im Hintergrund, trotz einiger Briefe von Gottsched, Bodmer, Sulzer und eines Schäfergedichts von Surland.

Der Briefwechsel mit Voltaire schien Haller so wichtig, daß er ihn schon früher, außerhalb der Korrespondenz-Edition, erscheinen ließ ${ }^{13}$.

Nach Haller ist Heinrich Maria von Leveling (1766-1828), Professor in Ingolstadt, offenbar der erste, der Briefe aus Hallers Feder ediert hat ${ }^{14}$. Aus dem Besitz seines Vaters besaß er acht Schreiben, datiert Bern 1775-1777. In seiner Schrift findet sich manches angesprochen, was auch spätere Editoren beschäftigt: Die Suche nach weiteren Teilen der Korrespondenz - eine Anzeige im Reichsanzeiger blieb ohne Antwort -, Hallers schwer lesbare Handschrift und die nicht immer leichte Erklärung der angesprochenen Tatsachen.

In der Mitte des 19.Jahrhunderts wird Hallers Korrespondenz für verschiedene Forscher und Schriftsteller wichtig. Herminie Chavannes benützt für die erste eigentliche historische Biographie Hallers die ihr in der Westschweiz zugänglichen Briefe ${ }^{15}$. Während bei ihr noch ein starkes erbauliches Moment zu verspüren ist, geht es dem Astronomen Rudolf Wolf 
(1816-1893) um die Wissenschaftsgeschichte. Er erwirbt sich besondere Verdienste, indem er Hallers Berner Briefsammlung auszugsweise veröffentlicht, erörtert und in seinen Biographien zur Kulturgeschichte der Schweiz als wertvolle Belege einbaut ${ }^{16}$.

Auch im Umkreis von Hallers früherer Wirkungsstätte wird man aktiv. E. Rössler benützt 1855 Briefe Hallers zur Beleuchtung der Gründungsgeschichte der Universität Göttingen ${ }^{17}$, E. Bodemann publiziert Material aus dem Nachlaß von J. G. Zimmermann in Hannover ${ }^{18}$. - Ischer veröffentlicht dazu 1905 die Gegenbriefe Zimmermanns nach den Manuskripten in der Stadtbibliothek Bern ${ }^{19}$.

Lange Zeit war es die Ausnahme, daß beide Partner einer Korrespondenz gleichwertig behandelt werden - Haller hat dem mit seinen Briefausgaben Vorschub geleistet. So haben Sigerist und Hintzsche (u. a. auch mit Rücksicht auf die Realisierbarkeit ihrer Vorhaben) mit Absicht darauf verzichtet, neben den Briefen Hallers auch die Gegenseite (J.Gessner, Morgagni, Caldani, Somis und Tissot) im Wortlaut wiederzugeben, obwohl sie diese kannten und benützt hatten. Einen Markstein in der Frage der Gleichberechtigung stellt H. Fischers Ausgabe des Briefwechsels von Haller mit E. F. v. Gemmingen dar ${ }^{20}$. - In neuester Zeit ermöglicht erst die großzügige Unterstützung der Burgergemeinde Bern und ihrer Albrecht von HallerStiftung so umfangreiche Briefwechsel wie den Hallers und Bonnets zu drucken.

Diese kurzen Bemerkungen lassen deutlich werden, daß es heute nicht leicht ist, den Überblick darüber zu gewinnen, welche Briefe sich wo befinden und was wo publiziert worden ist.

\section{Ein «Berner Haller-Projekt»}

Aus der Einsicht, daß die sich stellende Aufgabe der kritischen Bestandesaufnahme der Haller-Quellen die Kräfte von Einzelforschern bei weitem überfordert, entstand im Umkreis der Berner Burgerbibliothek, der Hüterin des Haller-Nachlasses, und der Albrecht von Haller-Stiftung der Burgergemeinde Bern ein Projekt, das die vordringlichsten Desiderata der HallerForschung verwirklichen soll ${ }^{21}$.

Das Ziel besteht in der Erschließung, Erforschung und Darstellung von Nachlaß, Leben und Werk Hallers, namentlich im Hinblick auf 
- das Netz seiner Korrespondenz,

- die wissenschaftliche Arbeit und Lektüre,

- das gedruckte Werk und die Rezensententätigkeit,

- seine Biographie (Chronik).

Den Ausgangspunkt bildet Hallers Nachlaß, die Sammlung seiner gedruckten Werke sowie der Bestand der Sekundärliteratur in der Burgerbibliothek Bern. Beigezogen werden sollen auch Halleriana in anderen Bibliotheken und Sammlungen.

Mit Rücksicht auf Struktur und Umfang der Haller-Quellen soll sich das Projekt in Einzelphasen mit gut definierbaren Zielen gliedern. Dabei ergeben sich charakteristischerweise zwei wesentliche Hauptaspekte, jener der Bestandeserschließung in der Burgerbibliothek und in anderen Sammlungen und jener der Forschung, z. B. wissenschaftliche Bearbeitung der Quellen bezüglich Entstehungsgeschichte, inhaltlichen Zusammenhang der Quellen, Bedeutung für Hallers Werk, Wirkung nach außen.

Neben der Phase «Hallers Korrespondenz», für die die Ausgangslage in diesem Artikel kurz skizziert ist, ist ein Phase vorgesehen, die sich mit seinen wissenschaftlichen Manuskripten und den etwa 10000 Urteilen über gelesene Bücher (Iudicia librorum) beschäftigt; auf diese soll eine Phase folgen, die Hallers gedruckte Werke und die über 10000 Rezensionen erschließt. Die Ergebnisse sollen phasenweise publiziert werden. Zum Schluß sollen die dafür geeigneten Elemente in einer Chronik zu Hallers Leben und Werk zusammengefaßt werden.

Die organisatorischen Vorbereitungen hat die Burgerbibliothek bereits 1988 an die Hand genommen, indem die erwähnte Neuordnung der Briefsammlung nach Korrespondenten durch Herrn Dr.E. Erne zugleich als Vorbereitungsphase für das Projekt dient. Weitere Abklärungen sind zur Zeit im Gang. Die Arbeiten der ersten, auf eine Dauer von vier Jahren geplanten Phase sollten gegen Ende 1990 aufgenommen werden können. 


\section{Anhang \\ Übersicht über die 66 Korrespondenten Hallers mit 30 und mehr Briefen in der Burgerbibliothek Bern (nach Thormann)}

\begin{tabular}{|c|c|c|}
\hline Anzahl Briefe & Korrespondent, Ort & Zeitl. Umfang \\
\hline 1585 & Werlhof, Paul Gottlieb, Hannover & $1736-1767$ \\
\hline 635 & Gessner, Johannes, Zürich & $1728-1777$ \\
\hline 563 & Münchhausen, Gerlach Adolf v., Hannover & $1736-1769$ \\
\hline 558 & Engel, Samuel, Bern & $1737-1777$ \\
\hline 485 & Tissot, Samuel Auguste A.D., Lausanne & $1754-1777$ \\
\hline 483 & Bonnet, Charles, Genf & $1754-1777$ \\
\hline 215 & Zimmermann, Joh. Georg, Brugg, Hannover & $1751-1777$ \\
\hline 183 & Somis, Ignazio, Turin & $1754-1777$ \\
\hline 167 & Saussure, Horace Bénédict de, Genf & $1760-1776$ \\
\hline 142 & Thierry, François, Paris & $1751-1777$ \\
\hline 136 & Zinn, Joh. Gottfried, Göttingen & $1750-1759$ \\
\hline 135 & Coppet, Abraham Louis de, Aigle & $1756-1777$ \\
\hline 122 & Ludwig, Christian Gottlieb, Leipzig & $1736-1772$ \\
\hline 115 & Gagnebin, Abraham, La Ferrière & $1739-1772$ \\
\hline 115 & Caldani, Leopoldo M. A., Bologna, Padua & $1756-1777$ \\
\hline 112 & Spielmann, Jakob Reinbold, Straßburg & $1753-1777$ \\
\hline 112 & Michaelis, Joh. David, Göttingen & $1751-1774$ \\
\hline 106 & Stettler, Joh. Friedrich, Bern & $1758-1777$ \\
\hline 105 & Beurer, Joh. Ambrosius, Nürnberg & $1745-1754$ \\
\hline 100 & Gmelin, Joh. Georg, Petersburg, Tübingen & $1725-1755$ \\
\hline 94 & Staehelin, Benedict, Basel & $1729-1748$ \\
\hline 83 & Pringle, John, London & $1760-1777$ \\
\hline 74 & Meckel, Joh. Friedrich, Göttingen, Berlin & $1744-1773$ \\
\hline 67 & Haller, Gottlieb Emanuel, Bern & $1752-1769$ \\
\hline 66 & Popowitz, Joh. Sigmund Valentin, Wien & $1750-1763$ \\
\hline 65 & Steiger, Franz Ludwig, Bern & $1736-1753$ \\
\hline 62 & König, Emanuel, Basel & $1732-1747$ \\
\hline 60 & Schreiber, Joh. Friedrich, Petersburg & $1728-1758$ \\
\hline 59 & Mieg, Achilles, Basel & $1755-1777$ \\
\hline 57 & La Chenal, Werner de, Basel & $1759-1777$ \\
\hline 57 & Herport, Gabriel, Bern & $1756-1764$ \\
\hline 54 & Housset, Etienne Jean Pierre, Auxerre & $1755-1774$ \\
\hline 51 & Engel, Jgfr., Unterseen & $1736-1753$ \\
\hline 51 & Murray, Joh. Philipp, Göttingen & $1751-1774$ \\
\hline 50 & Oeder, Georg Christian, Kopenhagen & $1751-1768$ \\
\hline 50 & Hugo, August Johann, Hannover & $1732-1764$ \\
\hline 50 & Heyne, Christian Gottlob, Göttingen & $1763-1777$ \\
\hline 48 & Wargentin, Peter, Stockholm & $1749-1777$ \\
\hline 48 & Ramspeck, Joh. Christoph, Basel & $1748-1763$ \\
\hline 48 & Grasset, François, Lausanne & $1756-1776$ \\
\hline 46 & Mylius, Christlob, Berlin & $1751-1754$ \\
\hline
\end{tabular}


Betrand, Elie, Bern

Tscharner, Vinzenz Bernhard, Bern

\section{Liste von Publikationen, in denen Briefe veröffentlicht werden}

Die Liste ist keineswegs vollständig. Sie gibt lediglich den gegenwärtigen Kenntnisstand eines Interessierten wieder, der die Sammlung der Burgerbibliothek Bern (BBB), des Berner Medizinhistorischen Instituts und die Bibliographien von Toellner und Zaunick benützt hat ${ }^{22}$. - Ergänzungen und Korrekturen sind jederzeit willkommen.

Die auszugsweise von Haller und R. Wolf publizierten Briefe sind hier nicht aufgenommen, ebensowenig Publikationen, in denen Briefe lediglich benutzt werden. - Ein detaillierter Nachweis der publizierten Briefe ist im Berner Haller-Projekt vorgesehen.

Asch: Siehe Sinner.

Baillod: Gerber-Visser, G., Die Briefe Abraham Baillods an AvH. Seminararbeit Univ. Bern, 1976 (BBB).

Bause: Thormann, F., Ein Brief AvHs [an den Graveur Bause in Leipzig]. Blätter f. bern. Gesch., Kunst u. Altertumskunde 17, 329-330, 1921.

Bernard: Hintzsche, E., AvHs Korrespondenz mit Johann Stephan Bernard. Clio medica 1, $324-340,1966$. 
Bianchi: Simili, A., Carteggio inedito di AH con Giovanni Bianchi (Jano Planco) ed appendice di altri inediti documenti halleriani. Minerva medica, Torino, 56, 241-258, 1965.

Bentinck: Geurts, A., Hesen, J., Poulisse, A. (éd.), Charlotte-Sophie Bentinck et AvH. Leur Correspondance inédite (1745-1768). LIAS (Sources and documents relating to the early modern History of Ideas) 6, 237-326, 1979 (BBB).

Boerhaave: Lindeboom, G.A., Boerhaave's Correspondence, part two, Leiden 1964, 20-23 (Boerhaave an Haller, 15.3.1736).

Bonnet: Sonntag, O. (Ed.), The Correspondence between AvH and Charles Bonnet, Bern, Stuttgart, Vienna 1983 (Studia Halleriana I).

Burggrave, J.P.: Boschung, U., Ein Brief AHs über seinen Aufenthalt in Paris und Straßburg (1728). Physis 19, 185-196, 1977.

Caldani: Hintzsche, E. (Hrsg.), AvH - Marc Antonio Caldani, Briefwechsel 1756-1776, Bern, Stuttgart 1966.

Crausaz: Hintzsche, E., Ein Brief AvHs über den Nutzen der Geburtszange. Schweiz. Ärztezeitung 39, 473-477, 1958 (Jean Daniel Crausaz an Haller, Haller an den bernischen Sanitätsrat).

Diesbach, v.: de Quervain, Th., Wie AHs Inschrift am Beinhaus in Murten entstanden ist. Berner Ztschr. f. Gesch. u. Heimatkunde, 163-164, 1950 (Brief Hs an Gottlieb von Diesbach, 1755).

Euler: Burckhardt, J. J., Vier Briefe von L[eonhard] Euler an AvH. Vjschr. d. Naturforsch. Ges. in Zürich 121, 363-366, 1976.

Gagnebin: Beer, G. R. de, Gagnebin, B. (éd.), Abraham Gagnebin de La Ferrière d'après sa correspondance. Bulletin de la Soc. neuchâteloise des sci. nat. 80, 45-79, 1957.

Gemmingen: Fischer, H. (Hrsg.), Briefwechsel zwischen AvH und Eberhard Friedrich von Gemmingen .... Tübingen 1899 (Bibliothek des Litterarischen Vereins in Stuttgart, CCXIX) (Reprint Königstein/Ts. 1979) (BBB).

Gessner: Sigerist, H.E. (Hrsg.), AvHs Briefe an Johannes Gesner (1728-1777), Berlin 1923 (Abhandlungen der Kgl. Gesellschaft der Wissenschaften zu Göttingen, math.-physikal. Klasse, N. F., Bd. XI, 2) - Nachträge von E. Hintzsche (Gesnerus 8, 98-113, 1951), E. Schmidt und W.Blasius (Sudhoffs Archiv 46, 251-263, 1962), U.Boschung (Bern 1972 und Gesnerus $31,267-287,1974)$.

Giller: Wegelin, C., Briefe des St. Galler Stadtarztes Peter Giller an AvH. Gesnerus 7, 1-26, 1950 .

Gmelin: Plieninger, G.H.T., Johannis Georgii Gmelini Reliquias quae supersunt commercii epistolici cum Carolo Linnaeo, Alberto Hallero, Guilielmo Stellero et al. ... publicandas curavit, Stuttgartiae 1861 (1 Brief von Gmelin an H, 24 Briefe Hs an Gmelin, 1743-1751). Haller-v. Haller, Emilie: siehe Zimmermann.

Herrenschwand: Briefe AvHs an den Nationalökonomen Jean Herrenschwand. Archiv d. Hist. Vereins d. Kts. Bern 26, 107-123, 1922.

Holstein-Gottorp: Strahlmann, B., AvH und Herzog Peter Friedrich Ludwig von Oldenburg, mit Briefen AvHs an die Prinzen von Holstein-Gottorp und an den Obersten von Staal. Berner Ztschr f. Gesch. u. Heimatkunde, 1958/3, 115-149.

Kästner, Abraham Gotthelf: Briefe aus sechs Jahrzehnten, 1745-1800, Berlin 1912 (Toellner). Klopstock: Betteridge, H.T., Klopstock's correspondence with AvH. Modern Language Review 58, 377-390, 1963. 
König: Wolf, R., Auszüge aus Samuel Königs Briefen an AvH. Mitth. d. Naturforsch. Ges. in Bern, 1845.

La Chenal: Christ, H., Der Briefwechsel der Basler Botaniker des 18. Jahrhunderts Achilles Mieg, Werner de La Chenal und Jacob Christoph Ramspeck mit AvH. Verh. Naturf. Ges. Basel XXIX, 1918.

Lambert: Steck, M., Johann Heinrich Lamberts Briefe an AvH. Nova Acta Leopoldina, N.F. 27, 7-18, 1963.

Leveling: Leveling, Heinrich Maria de (ed.), Epistolae Halleri ad Levelingium scriptae, quas edidit, praefatus est, notisque illustravit D. H' M' de L', filius, Erlangae 1795.

Linné: Smith, J.E., A selection of the Correspondence of Linnaeus and other naturalists from the original manuscripts, London 1821, Bd. 2, 228-437 (BBB). - Brief an Haller. Wiener Med. Wschr., 572-574, 1853 (Toellner); Lawrence, G.H.M., A letter by Carl Linnaeus to vH. Huntia 1, 166-168, 1964.

Ludwig: Singer, S., Ein ungedruckter Brief AvHs. Archiv f. d. Studium d. neueren Sprachen u. Literaturen 144 (N.S. 44), 250, 1923 (Zaunick) (Haller an C. G. Ludwig in Leipzig, 1750).

Maupertuis: Le Sueur, A., Maupertuis et ses correspondants. Lettres inédites. Correspondance de Maupertuis avec H, Montreuil-sur-Mer 1896 (Reprint Genève 1971), 180-204, 440-442 (BBB).

Michaelis: Ebstein, E., Aus der Frühzeit der Göttinger Gelehrten Anzeigen, mit einem unbekannten Brief I.D. Michaelis an AvH. In: Von Büchern und Menschen, Festschrift für Fedor von Zobeltitz, Weimar 1927.

Mieg, Achilles: siehe La Chenal.

Morgagni: Hintzsche, E. (Hrsg.), AvH - Giambattista Morgagni, Briefwechsel 1745-1768, Bern, Stuttgart 1964.

Münchhausen: siehe Sinner.

Muralt, v.: Kekulé v. Stradonitz, S., AvH und die Akademie der Arkadier zu Rom. Geisteskultur und Volksbildung 31, 58-63, 1922 (2 Briefe von Ludwig v. Muralt an AvH) (Zaunick).

Mylius: Geiger, L., Ein Brief von Christlob Mylius an AvH. Vjschr. Litteraturgesch. 3, 367-373, 1890 (Zaunick). - Cosentius, E., Briefe eines Berliner Journalisten. Euphorion, Ztschr. f. Literaturgesch. 10, 518-549, 1903 (Briefe von Christlob Mylius an AvH) (BBB).

Needham: Mazzolini, R.G., Two Letters on Epigenesis from John Turberville Needham to AvH. Journal of the Hist. of Med. All. Sci. 31, 68-77, 1976.

Nordenflycht: Stalmarck, T., Hedwig Charlotta Nordenflychts brev till vH och Johan Arckenholtz. Samlaren, Tidskrift for svensk litteraturhistorisk forskning, Uppsala, N.F. 40, 106-121, 1959.

Pontedera: Peruzzi, D., Tre lettre di Guilio Pontedera ad AdH. Atti e Mem. Accad. Stor. Arte sanit. (IIA) 23 71-74, 1957 (BBB).

Ramspeck, Jacob Christoph: siehe La Chenal.

Rast: Vernay, Correspondance inédite d'AdH, de Barthèz, Tronchin, Tissot avec le docteur Rast, de Lyon. Lyon 1856 (BBB); auch in Gaz. méd. de Lyon 8, 1, 21, 45, 69, 145, 161, 1856 (Toellner).

Réaumur: Pelseneer, J., Une lettre inédite de Réaumur à H. Bull. Acad. roy. Belgique, Cl. Sci. 5e série, 37, 792-795, 1951 (BBB).

Rosén: Berg, F., Correspondence between Nils Rosén von Rosenstein and AvH. Acta paediatrica (Uppsala), Suppl. ad Vol. 156, 103-139, 1964. 
Saussure, Horace-Bénédict de: in Vorbereitung.

Schobinger: Wegelin, C., Briefe des St.Galler Arztes David Christoph Schobinger an AvH. Gesnerus 8, 216-235, 1952.

Seligmann: Hintzsche, E., Politischer Nachrichtendienst in AvHs Korrespondenz. Berner Ztschr. f. Gesch. u. Heimatkunde, 41-46, 1958 (vier Briefe an J. M. Seligmann, Nürnberg).

Sinner: Rössler, E.F., Die Gründung der Universität Göttingen, Entwürfe, Berichte und Briefe der Zeitgenossen, Göttingen 1855: (Briefe Hs an Joh. Rud. Sinner, Landvogt von Saanen, G.Th. v. Asch, Heyne; an H von G.A. v. Münchhausen) - Siehe auch: Zimmermann. Somis: Hintzsche, E. (Hrsg.), AvH - Ignazio Somis, Briefwechsel 1754-1777, Bern, Stuttgart 1965. - Realini, Lucio, Carteggio fra Ignazio Somis e AH (1754-1764), Diss. med., Bern 1968; Pagnamento, Fausto, Carteggio fra Ignazio Somis e AH (1764-1777), Diss. med., Bern 1969. Spallanzani: Di Pietro, P., Carteggio fra Spallanzani ed H.Atti della Soc. dei Naturalisti e Matematici di Modena 92, 32-110, 1961.

Spielmann: Vetter, C., Strasbourg et l'Europe des Lumières, Lettres de Jacques Reinbold Spielmann à AvH, 1753-1777, Thèse en vue du doctorat, Université des Sciences Humaines de Strasbourg, 1986, 2 Bde., Typoskript.

Staal, v.: siehe Holstein.

Steiger: Bloesch, H., AH als Bibliothekar. In: Mélanges offerts à M. Marcel Godet, Bern 1937 (BBB) (Isaak Steiger an AH, 1736).

Swieten, van: Retzer, v., Briefwechsel zwischen Herrn von Haller und Herrn van Swieten. Neues Magazin für Ärzte, hrsg. von E.G. Baldinger, 2, 206-215, Leipzig 1780.

Tenon: Huard, P., Imbault-Huard, M.-J., Trois lettres de René Tenon à AdH. Hist. sci. méd. $12,397-407,1978$.

Thierry: Hamon, O., Contribution à l'étude des correspondants de Haller et en particulier de Thierry. Rennes 1970, thèse de médecine.

Thoms: Hintzsche, E., Boerhaaviana aus der Burgerbibliothek Bern. In: Lindeboom, G. A. (ed.), Boerhaave and his time, Leiden 1970, 144-164 (Briefe von Friedrich (v.) Thoms an AvH).

Tissot: Hintzsche, E. (Hrsg.), AvHs Briefe an Auguste Tissot, Bern, Stuttgart, Wien 1977. Minder-Chappuis, G., Auguste Tissot, sa correspondance et ses œuvres durant la période de 1754 à 1761. Thèse méd., Berne 1973.

Tscharner: Hamel, R. (Hrsg.), Briefe von J.G. von Zimmermann, Wieland und AvH an Vinzenz Bernhard von Tscharner, Rostock 1881, 61-83.

Voltaire: Briefe des Herrn von Voltaire mit den Antworten [Hallers]. In: [Haller, A. v., Hrsg.,] Sammlung kleiner Hallerischer Schriften, Bern 1772, Bd.3, 353-373. - Dübi, H., Der Briefwechsel zwischen Voltaire und Haller im Jahre 1759. Archiv f. d. Studium d. neueren Sprachen und Literaturen 123, 353-386, 1910.

Werlhof: Frensdorf, F., Briefe zweier hannoveranischer Ärzte (P.G. Werlhof u. J.G.Zimmermann) an AvH. Ztschr. d. hist. Vereins f. Niedersachsen 103-198, $1891{ }^{23}$.

Wolff: Schuster, J., Der Streit um die Erkenntnis des organischen Werdens im Lichte der Briefe C.F. Wolffs an AvH. Sudhoffs Archiv Gesch. Med. 34, 196-218, 1941. - Belloni, L., Embryological drawings concerning his Theorie von der Generation sent by Caspar Friedrich Wolff to AvH in 1764. Journal of the hist. of med. and all. sci. 26, 205-208, 1971. - Roe, S., Matter, Life, and Generation. Eighteenth-century embryology and the Haller-Wolff debate, Cambride (etc.) 1981, S. 158-173 (engl. Übersetzung). 
Zimmermann: Bodemann, E. (Hrsg.), Von und über AvH, Hannover 1885 (Briefe Hs an J.G.Zimmermann, J. R.Sinner, Emilie Haller-v. Haller). - Ischer, R. (Hrsg.), J.G.Zimmermanns Briefe an H. Nach dem Manuskript der Stadtbibliothek Bern. Neues Berner Taschenbuch auf das Jahr 1904 [-1912], Bern 1903-1911. - Reber, B., Lettres inédites des célèbres médecins Tissot et Zimmermann. Bibliothèque historique de la France médicale, No.42, Paris 1912 (Zimmermann an Haller, 30.6.1760). - Siehe auch: Werlhof.

\section{Anmerkungen}

1 Hirzel, L., Albrecht von Hallers Gedichte, hrsg. u. eingeleitet, Frauenfeld 1882 (Bibliothek älterer Schriftwerke der Schweiz, 3. Bd.).

2 Die Mailänder Haller-Bestände sind aufgearbeitet; für die Manuskripte siehe: Pecorella Vergnano, L., Il fondo Halleriano della Biblioteca Braidense di Milano, Milano 1965 (Istituto di Storia della Medicina, Studi e testi 8); Nachtrag bei Monti (siehe unten), Parte I, Vol. III, tom. II. - Für Hallers Bibliothek siehe: Monti, M. T., Catalogo del Fondo Haller della Biblioteca Nazionale Braidense di Milano. Milano, Franco Angeli Editore (Filosofia e scienza nel Cinquecento e nel Seicento, Pubblicazioni del «Centro di studi del pensiero filosofico del Cinquecento e del Seicento in relazione ai problemi della scienza» del Consiglio Nazionale delle Ricerche), 1983 ff.: Parte I: Libri: Vol.I, II, III.1, III.2 (1983-1984); Parte II: Dissertazioni: Vol. I-V (1985-1987).

3 Thormann, F., Register der Briefsammlung von AvH in der Berner Stadtbibliothek. Bern (1935), 24 S. (Beilage zum Bericht der Stadtbibliothek über die Jahre 1933-1935). Die Geschichte des Verkaufs und der Rückkehr von Hallers Bibliothek sowie Beschreibung der in Mailand verbliebenen Manuskripte siehe Pecorella Vergnano (Anm.2). - Den Berner Bestand verzeichnet Boschung, U., Kurzinventar der Manuskripte Albrecht von Hallers [in der Burgerbibliothek Bern], Bern 1974 (Typoskript).

4 Burgerbibliothek Bern, Mss. hist. helv. XVIII 68.

5 Thormann (Anm.3), S.2 (Vorwort von H.Bloesch). - Nach den angegebenen Zahlen würde der Verlust 942 Briefe betragen, wobei von 95 Korrespondenten sämtliche Briefe verschwunden sind.

6 Eine von Dr. H. Haeberli, Leiter der Burgerbibliothek bis 1988, angelegte Kartei gibt darüber Auskunft.

7 Die Serien trugen in der Berner Stadtbibliothek und in der Burgerbibliothek vor der Auflösung der Bände folgende Signaturen:

Mss. hist. helv. XVIII 1-38

Mss. hist. helv. XVIII 39-41

Mss. hist. helv. XVIII 42-53

38 Bände, 1724-1777;

3 Bände, 1726-1775;

12 Bände, 1736-1778.

Die Briefe von G.A. v. Münchhausen an Haller trugen die Signatur:

Mss. hist. helv. XVIII 62-64

3 Bände, 1736-1761.

Die Briefe Paul Gottlieb Werlhofs an Haller trugen die Signatur:

Mss. hist. helv. XVIII 54-61

8 Bände, 1736-1758.

Die von der Burgerbibliothek gesammelten Briefe Hallers an verschiedene Korrespondenten tragen die Signatur:

Mss. hist. helv. XVIII 78 (Name der Korrespondenten). 
8 Thormann (Anm. 3), S.2.

9 Schmidt-Herrling, E., Die Briefsammlung des Nürnberger Arztes Christoph Jakob Trew (1695-1796) in der Universitätsbibliothek Erlangen, Erlangen 1940, S. 246-248.

10 Hallers Vorwort zu «Epistolarum ...» (Anm.11), Bd.6, S.III: Bartholin, Thomas, Epistolarum medicinalium a doctis vel ad doctos scriptarum centuria I (-IV), Kopenhagen 1663-1667 (und spätere Editionen). Vgl. Bibl. anat. II, 405: «Grata hic varietas, neque certe sterilis.»

11 Epistolarum ab eruditis viris ad Alb. Hallerum scriptarum Pars I. Latinae, Bernae 1773-1775, 6 Bände. - Verteilung der Briefe: Bd.1: Nr. 1-194; Bd. 2: Nr. 195-404; Bd.3: Nr. 405-670; Bd.4: Nr. 1-132; Bd. 5: Nr. 133-277; Bd. 6: Nr. 278-334.

12 Einiger gelehrter Freunde deutsche Briefe an den Herrn von Haller. Erstes Hundert von 1725 bis 1751, Bern 1777.

13 Briefe des Herrn von Voltaire mit den Antworten [Hallers]. In: Sammlung kleiner Hallerischer Schriften, Bern 1772, Bd. 3, 353-373.

14 Leveling, H.M. de (ed.), Epistolae Halleri ad Levelingium scriptae, quas edidit, praefatus est, notisque illustravit D. H'M' de L', filius, Erlangae 1795.

15 (Chavannes, H.), Albert de Haller, Biographie, Lausanne 1840, seconde édition, Paris 1845 (Briefe Hs an Tissot und Bonnet benützt).

16 Wolf, R., Auszüge aus Samuel Königs Briefen an AvH, mit litterarisch-historischen Notizen, Mitth. d. naturforsch. Ges. in Bern, Bern 1845; ders., [220] Auszüge aus Briefen an AvH, ebd. 1846-1848; ders., Biographien zur Kulturgeschichte der Schweiz, Zürich 1858-1862, 4 Bde.

17 Rössler, E.F., Die Gründung der Universität Göttingen, Entwürfe, Berichte und Briefe der Zeitgenossen, Göttingen 1855 (Briefe Hs an Landvogt Sinner von Saanen, G.Th. v. Asch, Heyne; an H von G. A. v. Münchhausen).

18 Bodemann, E., Von und über AvH, Hannover 1885 (Briefe Hs an J.G.Zimmermann, J. R.Sinner, Emilie Haller-v. Haller).

19 Ischer, R. (Hrsg.), J.G.Zimmermanns Briefe an H. Nach dem Manuskript der Stadtbibliothek Bern. Neues Berner Taschenbuch auf das Jahr 1904 [-1912], Bern, 1903-1911.

20 Fischer, H. (Hrsg.), Briefwechsel zwischen AvH und Eberhard Friedrich von Gemmingen ..., Tübingen 1899 (Bibliothek des Litterarischen Vereins in Stuttgart, CCXIX) Reprint Königstein/Ts. 1979) (BBB).

21 Den Ausführungen liegt ein vom Verfasser und den Herren Dr. H. Haeberli (Leiter der Burgerbibliothek bis Ende 1988) und lic.phil. J.H. Wäber (Konservator der neueren Handschriften der Burgerbibliothek seit 1989) erarbeitetes Konzept zugrunde.

22 Toellner, R., Albrecht von Haller, Über die Einheit im Denken des letzten Universalgelehrten, Wiesbaden 1971 (Sudhoffs Archiv, Beiheft 10), S.203-228. - Zaunick, R., Supplement zu J.C.Poggendorff, Biogr.-lit. Handwörterbuch der exakten Naturwissenschaften, Bd. VII a, Berlin 1971, S. $254 \mathrm{f}$.

23 Hallers Briefe an Werlhof konnten trotz einer 1981 im Auftrag der Albrecht von HallerStiftung der Burgergemeinde Bern von Dr. Horst Meyer, Wolfenbüttel, vorgenommenen Archivreise nicht gefunden werden. 


\section{Summary}

\section{Working up Albrecht von Haller's correspondence}

During his whole life, Albrecht von Haller (1708-1777) collected the letters sent to him by his scientific correspondents. These letters are conserved by the Burgerbibliothek Bern. Thormann's inventory of $1933 / 35$ naming 1114 correspondents with a total of 12260 letters is shortly analyzed and a project for a new scientific inventory and investigation of Haller's manuscripts is outlined.

\section{Résumé}

\section{Les travaux sur la correspondance d'Albert de Haller}

La correspondance scientifique collectionnée par Albert de Haller (1708-1777) est conservée par la Bibliothèque de la Bourgeoisie de Berne (Burgerbibliothek). La liste de 1933/35, établie par F. Thormann, nomme 1114 correspondants et 12260 lettres. L'auteur donne une brève analyse de cette liste; il présente les bases de départ et les idées directrices pour un projet d'inventaire scientifique des manuscrits de Haller.

Prof. Dr. med. Urs Boschung

Medizinhistorisches Institut der Universität

Bühlstraße 26

Postfach

CH-3000 Bern 9 